Journal of trends in Computer Science and Smart technology (TCSST) (2020)

Vol.02/ No. 02

Pages: $78-88$

https://www.irojournals.com/tcsst/

DOI: https://doi.org/10.36548/jtcsst.2020.2.002

\title{
A Comparative Performance Analysis of Fog-Based Smart Surveillance System
}

\section{Sujan Shrestha,}

Nepal Open University, Lalitpur, Nepal

Email:ersujan@gmail.com

Subarna Shakya,

Pulchowk Campus, Institute of Engineering,

Tribhuvan University, Lalitpur, Nepal

Email:drss@ioe.edu.np

\begin{abstract}
There has been increasing demand of security and safety in public as well as private places and hence surveillance system using IoT sensors, cameras has become the most important part in our daily life. This system has to operate all times 24/7/365 and thus produces huge amounts of data. Cloud computing offers storage, processing, and analytical services for handling of such massive amounts of data. For real time applications like smart surveillance system, increased latency from centralized Cloud computing is not acceptable. Fog Computing is an extension of Cloud computing, evolved to minimize latency. A Fog-Based Smart Surveillance System has been modelled and simulated in two environments as Cloud Only Network and Fog-Based Cloud Network using iFogsim. Various performance metrics like Application Loop Delay, Energy Consumption, Execution Cost, and Network Usage has been compared between Fog-Based Cloud Network and Cloud Only Network. Results showed that Fog-Based Cloud Network performs better than Cloud Only Network.
\end{abstract}

Keywords: Fog Computing, Smart Surveillance System, IoT, Application Loop Delay, Energy Consumption, Execution Cost, Network Usage, iFogSim

\section{Introduction}

During the last few years, we have witnessed that the two major key technologies like the smart phone, cloud computing has revolutionized the way people communicate and interact each other and with machine, and added tremendous level of comforts in their lives. The main cause of success goes mainly to the introduction of cloud computing system that aid in reducing the burden of computing and storage capacity required for end user devices (Bellavista et al., 2019). In parallel, we also have witnessed the development and implementation of the Internet of Things (IoT) into our daily lives, in areas such as transportation, health care, agriculture, emergency response, industrial automation, home automation(Yousefpour, Ishigaki, \& Jue, 2018).

Cloud computing is the base stone for offering infrastructure, platform, and software services to develop IoT enabled systems that ultimately meets the requirement of future IoT applications(Buyya, 2018). However, there still exists some issues and challenges in traditional centralized cloud paradigm that needs to be solved by new cloud paradigm. In centralized cloud system, the cloud data centre usually resides remotely from end user devices which leads to higher latency. Besides, handling massive amount of raw data from massive amount of IoT devices add more cost due to the requirement of higher computing and storage capacity of cloud servers and more network bandwidth 
Journal of trends in Computer Science and Smart technology (TCSST) (2020)

Vol.02/ No. 02

Pages: $78-88$

https://www.irojournals.com/tcsst/

DOI: https://doi.org/10.36548/jtcsst.2020.2.002

requirement to the cloud servers. Fog computing is a promising solution to address above challenges in the cloud based IoT.

Fog Computing is a new paradigm in cloud computing that does not eliminate the use of centralized cloud services but rather extends the cloud to the edge of network and brings the cloud resources closer to the IoT devices, physically and/or computationally by increasing the layers in between cloud and end user devices(Shi et al., 2018). Fog Computing works as a middle layer between Cloud and end user IoT devices. In fog computing, the big data generated by different types of IoT devices and applications can be processed at the network edge instead of sending them all to the centralized cloud infrastructure thus reducing computation overload of cloud server, saving network bandwidth, reducing response time or latency and response location awareness. The cloud network in which all the modules of an application run only on a centralized cloud data centres is termed as Cloud only network and the cloud network where most of the modules (delay sensitive) of an application run on edge devices like router, switches, cellular base stations etc., near or close to IoT devices and some of the modules (delay tolerant) run on a centralized cloud data centre is termed as Fog-Based Cloud Network. Fog Computing is also sometimes termed as Edge Computing, Mobile Edge Computing, Cloudlets. Fog computing-based architecture can also be considered as the future of IoT infrastructure.

Mostly Sense Process Actuate Model (SPAM) (Jamil et al., 2020) is commonly used in Fog Computing applications. In this SPAM model, fog device gets data from sensors and does processing. Fog device sends results to actuator after processing to make necessary actions. Fog Devices also forward some data to Cloud for managing storage and processing for long term analytics and also if fog devices are unable to handle tasks due to limitation in resource. These fog computing applications are developed as Directed Acyclic Graph (DAG) that contains different modules following Distributed Data Flow (DDF) model. In DAG, a module takes input, processes it, and sends to output to another module as input. Smart mobile phones, cellular base stations, switches, routers, etc., can serve as fog devices. These fog devices are resource constrained in terms of processing capability, storage capacity, and limited power.

\section{Related Works}

Fog Computing is still in its infancy stage. Currently there are several research works underway focused on improving various performance metrics of the Fog-Cloud Network environment. A discussion about applications, issues, architecture, and protocols of fog computing have been presented in(Dastjerdi, Gupta, Calheiros, Ghosh, \& Buyya, 2016) .Several performance metrics for evaluating fog computing networks can be found in the literature of (Mahmoud et al., 2018), (Rahbari \& Nickray, 2019), and (Nakamura, Duolikun, Oma, Enokido, \& Takizawa, 2018) . They include power consumption, latency, response time, energy cost, and network utilization. Fog computing is at the nascent stage of development and adoption, and various simulation tools are recently is use in order to test and validate various technical solutions, resource management policies, empirical analysis and research works in Fog Computing. In (Svorobej et al., 2019), the author has reviewed about various Fog modelling and simulation tools, their comparative study, problems posed by fog computing in relation to simulation.

Out of various available simulation tools (Byrne et al., 2017), iFogSim simulator has gained remarkable attention from many Fog Computing researchers. The paper in (Buyya, 2018) has 
Journal of trends in Computer Science and Smart technology (TCSST) (2020)

Vol.02/ No. 02

Pages: $78-88$

https://www.irojournals.com/tcsst/

DOI: https://doi.org/10.36548/jtcsst.2020.2.002

discussed in details about various components of iFogSim simulator, installation procedures and example scenarios and its corresponding code snippet. In (Sucharitha, Prakash, \& Iyer, 2019),, authors has modelled and simulated IoT-Fog based farm management system using iFogsim and performed a comparative study considering performance metrics like Total Network usage and Average Latency of Control Loop in various configurations.

\section{Proposed Work}

The Fog-Based smart surveillance system application is designed and simulated in iFogsim to perform a comparative study on the performance of the Fog-Based Cloud Network and Cloud Only implementation. For better accuracy in comparison results, 5 different configuration has been used. The simulation environment in this research work is done in a personal computer with Intel Core i75600U CPU @2.6GHz, 8 GB RAM, OS as Microsoft Windows 10 64-bit using iFogsim . Eclipse IDE Version: 2020-03 (4.15.0), Build id: 20200313-1211 is used as Java IDE for this research project.

\section{A. Devices and Setup}

The Figure 1 shows a general hierarchical structure of the Smart Surveillance System topology created using the GUI module in iFogsim. The topology consists of the devices as Cloud Data Center, Proxy Server, Fog Device (Router), Cameras, Sensors, and Pan-Tilt zoom (PTZ) Actuators.

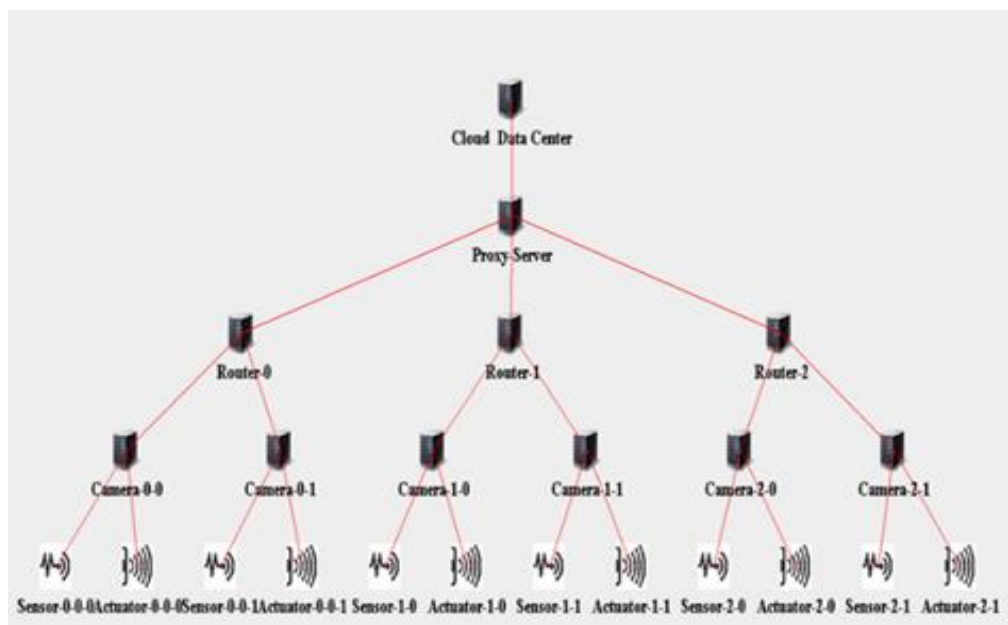

Figure 1Hierarchical Topology of Fog-Based Surveillance System

The Figure 2 (Gupta, Vahid Dastjerdi, Ghosh, \& Buyya, 2017) shows the application model of the Smart Surveillance System.The workflow loop in this system is as Camera >>MotionDetector >>ObjectDetector>>ObjectTracker\& User Interface >>PTZControl. 
Journal of trends in Computer Science and Smart technology (TCSST) (2020)

Vol.02/ No. 02

Pages: $78-88$

https://www.irojournals.com/tcsst/

DOI: https://doi.org/10.36548/jtcsst.2020.2.002

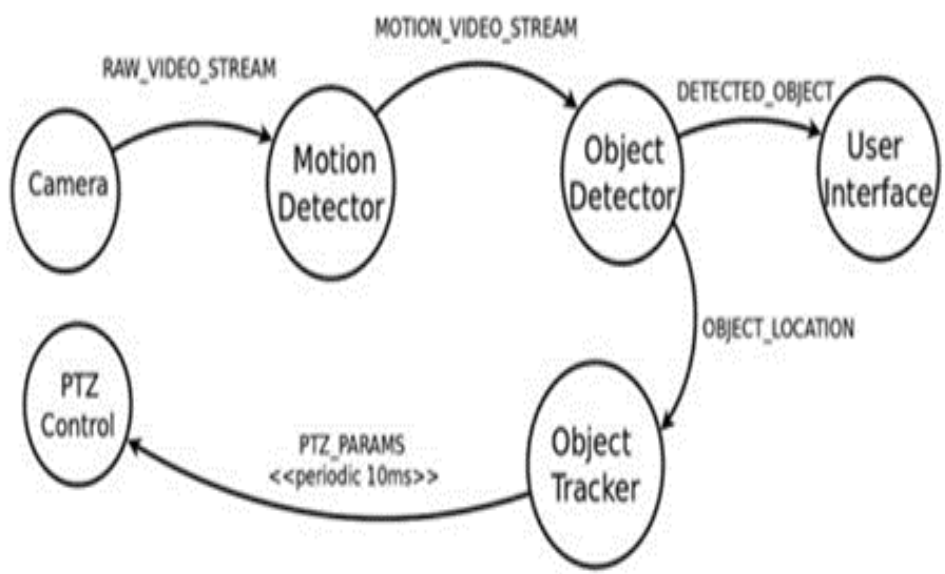

Figure 2Application model of the Smart Surveillance System

\section{B. Configurations}

The capabilities and the configuration of each devices, are given in the Table I. The latency of the connection between the devices are given in Table II. These configuration values are determined from the case in(Gupta et al., 2017).

Table ITopology configuration settings of devices

\begin{tabular}{|c|c|c|c|c|c|c|c|c|}
\hline$\frac{\mathscr{0}}{\frac{1}{\pi}}$ & $\frac{\cong}{\bar{\Sigma}}$ & 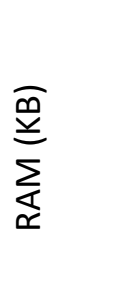 & 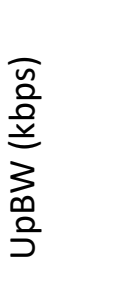 & 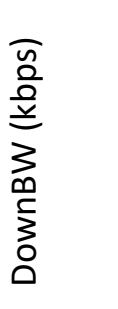 & $\overline{\mathrm{d}}$ & 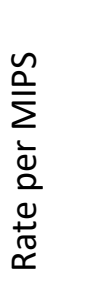 & 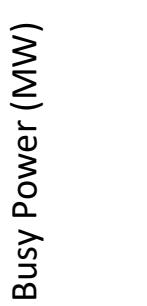 & 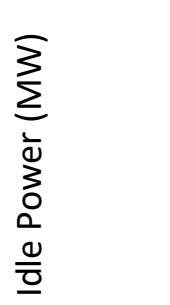 \\
\hline Cloud & 44800 & 40000 & 100 & 10000 & 0 & 0.01 & 1648 & 1.332 \\
\hline $\begin{array}{l}\text { Proxy- } \\
\text { Server }\end{array}$ & 2800 & 4000 & 10000 & 10000 & 1 & 0 & 107.339 & 83.4333 \\
\hline $\begin{array}{c}\text { Fog } \\
\text { Device }\end{array}$ & 2000 & 4000 & 10000 & 10000 & 2 & 0 & 107.339 & 83.4333 \\
\hline Camera & 500 & 1000 & 10000 & 10000 & 3 & 0 & 87.53 & 82.44 \\
\hline
\end{tabular}

Table IILatency Configuration

\begin{tabular}{|l|l}
\hline Connection & Latency (ms) \\
\hline
\end{tabular}


Journal of trends in Computer Science and Smart technology (TCSST) (2020)

Vol.02/ No. 02

Pages: $78-88$

https://www.irojournals.com/tcsst/

DOI: https://doi.org/10.36548/jtcsst.2020.2.002

\begin{tabular}{|l|l|}
\hline Cloud - Proxy Server & 100 \\
\hline $\begin{array}{l}\text { Proxy Server - Fog } \\
\text { Device }\end{array}$ & 2 \\
\hline Fog Device - Camera & 2 \\
\hline Camera - Sensor & 1 \\
\hline Camera - Actuator & 1 \\
\hline
\end{tabular}

The 5 different configuration used are named as Config 1, Config 2, Config 3, Config 4, and Config 5 . Config 1, Config 2, Config 3, Config 4, and Config 5 has 1, 2, 3, 4, and 5 surveillance area(s) respectively. The details of different configuration is listed in Table III. Each surveillance area means a fog device (router). Each fog device or each surveillance areas contains two PTZ Controller camera. The smart camera is modeled as video sensor and actuator node is modeled as the PTZ control feature of camera. The topology diagram of Config. 1 and Config. 5 configuration is shown in Figure 3 and 4.

Table IIIConfiguration Details

\begin{tabular}{|c|c|c|c|}
\hline $\begin{array}{c}\text { Config. } \\
\text { No. }\end{array}$ & $\begin{array}{c}\text { No of } \\
\text { Surveillance } \\
\text { Areas } \\
\text { (No. of Fog } \\
\text { Device/Rout } \\
\text { er) }\end{array}$ & $\begin{array}{c}\text { No of } \\
\text { cameras in a } \\
\text { surveillance } \\
\text { area }\end{array}$ & $\begin{array}{c}\text { Total No of } \\
\text { cameras }\end{array}$ \\
\hline 1 & 1 & 2 & 2 \\
\hline 2 & 2 & 2 & 4 \\
\hline 3 & 3 & 2 & 6 \\
\hline 4 & 4 & 2 & 8 \\
\hline 5 & 5 & 2 & 10 \\
\hline
\end{tabular}


Journal of trends in Computer Science and Smart technology (TCSST) (2020)

Vol.02/ No. 02

Pages: $78-88$

https://www.irojournals.com/tcsst/

DOI: https://doi.org/10.36548/jtcsst.2020.2.002

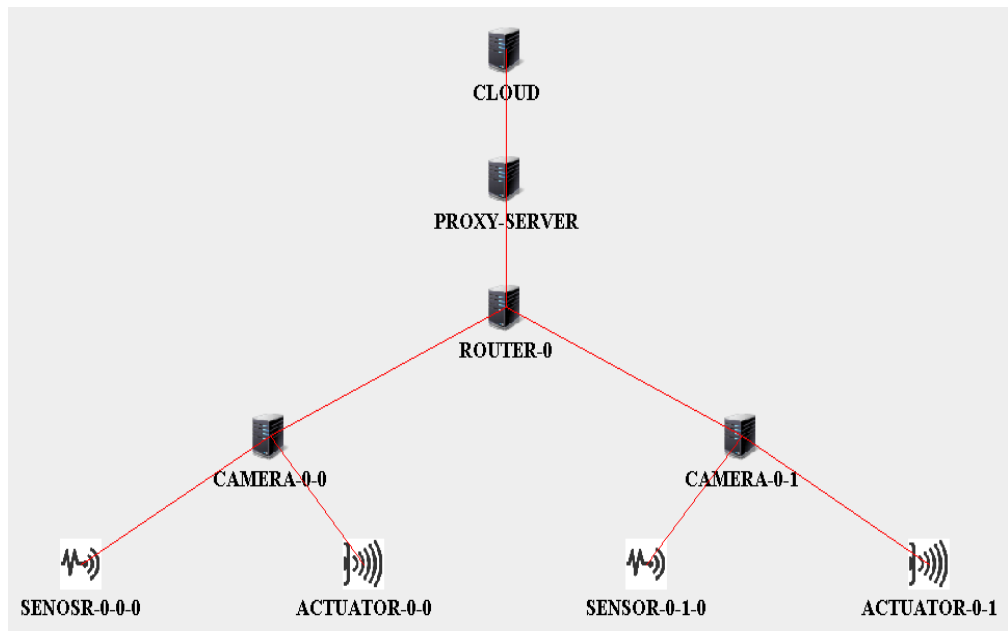

Figure 3Smart Surveillance System for Config 1

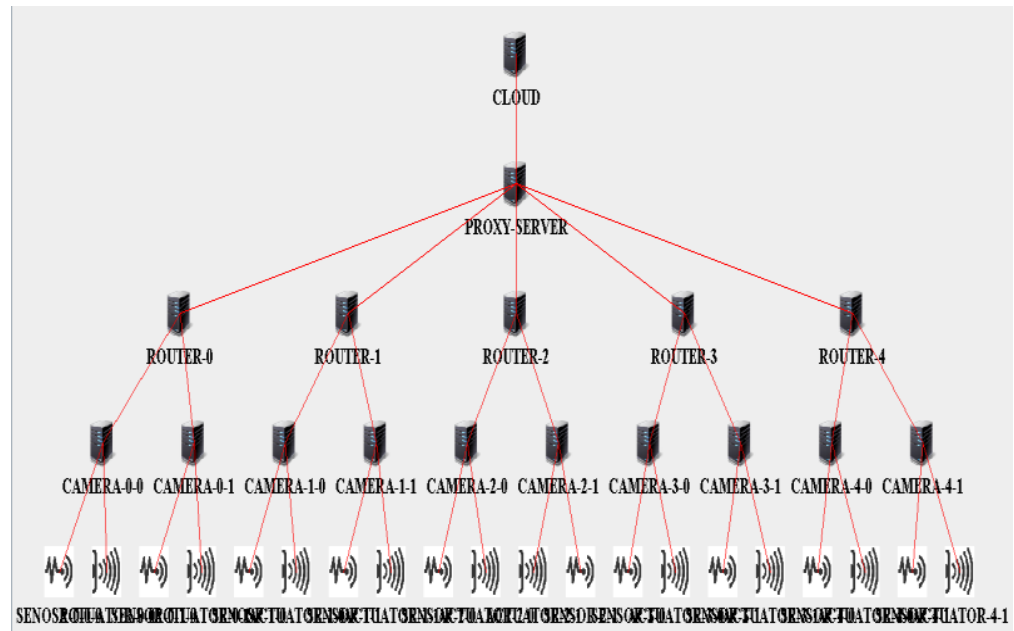

Figure 4Smart Surveillance System for Config 5

\section{Performance Metrics Calculation}

The performance metrics used in fog computing are Application Loop Delay, Energy Consumption, Cost of Execution, Network Usage, whose calculation are done as explained in (Jamil et al., 2020),(Rahbari \& Nickray, 2019).

\section{Results and Discussion}

The simulation results include the value of performance metrics like Application Loop Delay, Energy consumption by devices, Execution cost in cloud, and network usage. The plots and graphs of the various results are shown in Figure 5, 6, 7, and 8. 
Journal of trends in Computer Science and Smart technology (TCSST) (2020)

Vol.02/ No. 02

Pages: $78-88$

https://www.irojournals.com/tcsst/

DOI: https://doi.org/10.36548/jtcsst.2020.2.002

A. Application Loop Delay

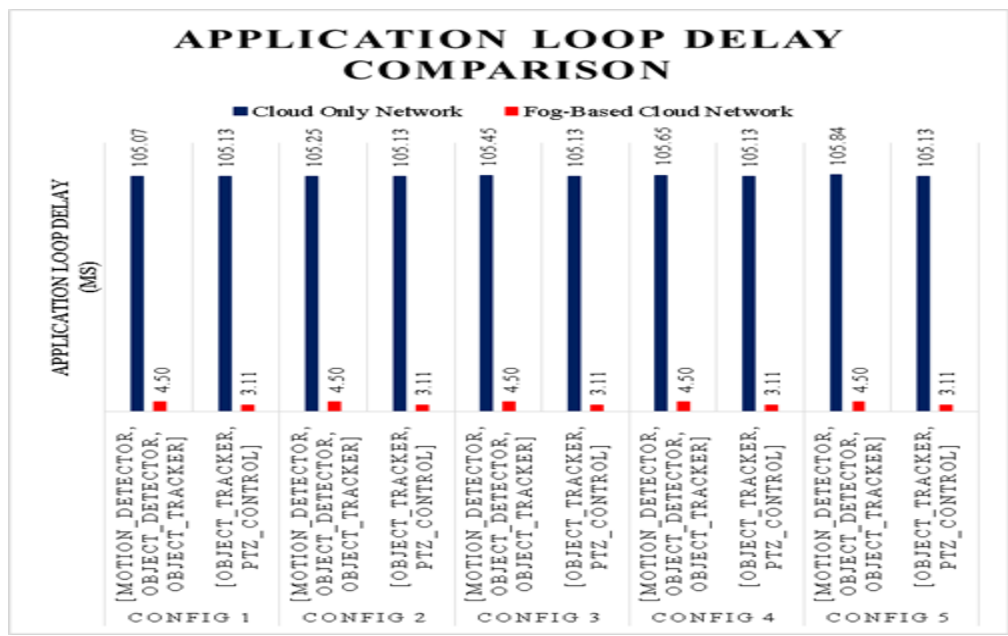

Figure 5Application Loop Delay Comparison

It is observed from Figure 5 that the both application loop delays from Source module motion_detector to desination module object_detector and then to destination module object_tracker, and from source module object_tracker to Destination module PTZ_Control are very much lesser in Fog-Based cloud network than that in Cloud Only Network in all 5 configurations. This result can be justified from the fact that in Cloud only network all the data are sent to the cloud data centre for processing and analysing while in Fog-Based Cloud network most of the data are processed and analysed at the fog devices and some data are sent to the cloud when the fog devices are unable to handle tasks or job due to its resource constraints. Minimization in application loop delay helps application more responsive and work faster.

B. Energy Consumption

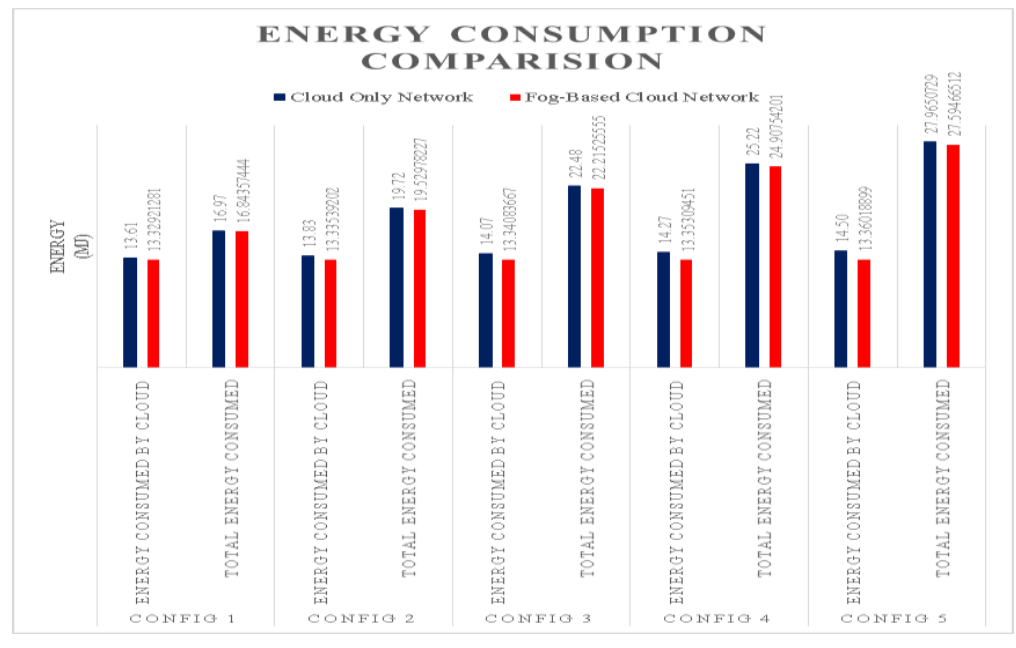

Figure 6Energy Consumption Comparison

It is observed from Figure 6 that the energy consumed by cloud and total energy consumption by all devices in a network are lesser in Fog-Based cloud network than that in Cloud Only Network in all 5

ISSN: 2582-4104 
Journal of trends in Computer Science and Smart technology (TCSST) (2020)

Vol.02/ No. 02

Pages: $78-88$

https://www.irojournals.com/tcsst/

DOI: https://doi.org/10.36548/jtcsst.2020.2.002

configurations. This result can be justified from the fact that burden of processing and analysing the data are more in Cloud only network than that in Fog-Based Cloud network. Reduction of energy consumption helps to cut down the operating cost of the IoT applications.

C. Cost of Execution in Cloud

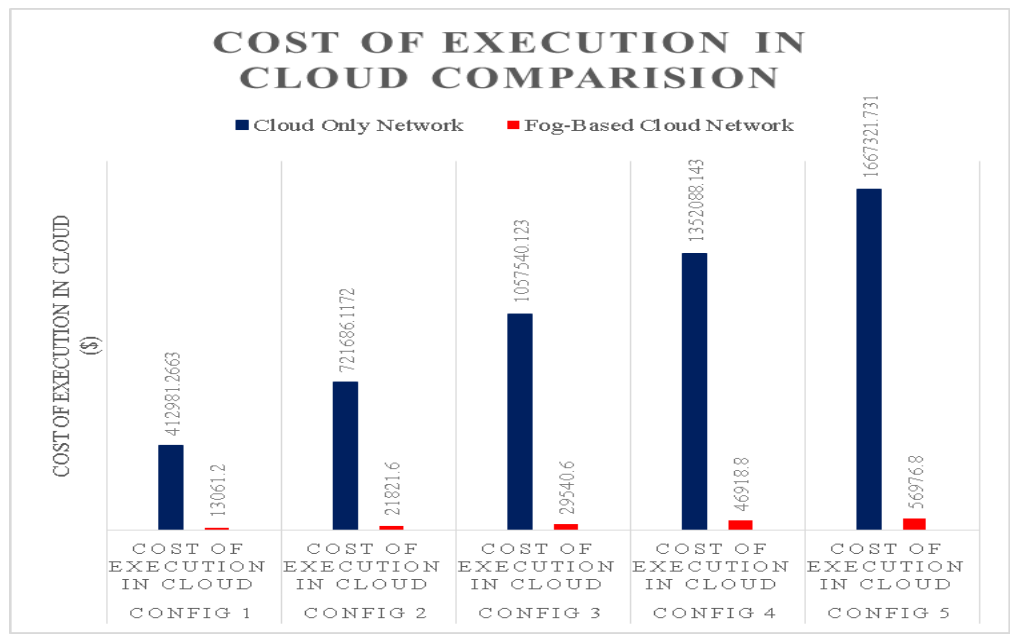

Figure 7Cost of Execution in Cloud Comparison

It is observed from Figure 7 that the cost of execution in Fog-Based cloud network is very much lesser than that in Cloud Only Network in all 5 configurations. This result can be justified from the fact that burden of processing and analysing the data in cloud data centre is reduced which ultimately reduced total energy consumption in Fog-Based Cloud. Reduction in execution cost helps to minimize budget for the large scale implementation of IoT applications in diverse sectors.

D. Total Network Usage

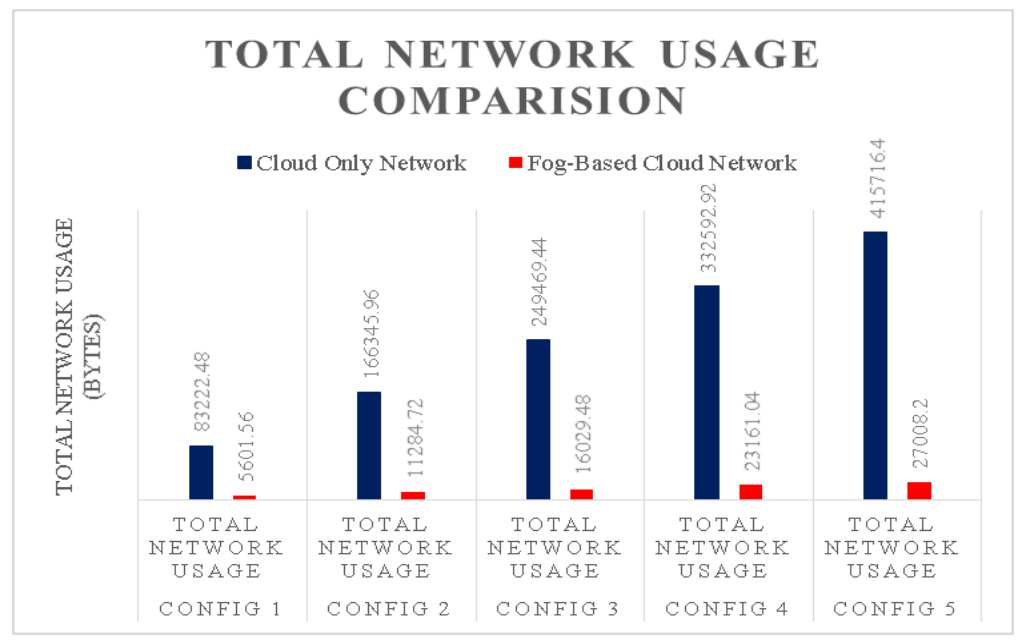

Figure 8Total Network Usage Comparison

It is observed from Figure 8 that network usages in Fog-Based cloud network is very much lesser than that in Cloud Only Network in all 5 configurations. This result can be justified from the fact that 
Journal of trends in Computer Science and Smart technology (TCSST) (2020)

Vol.02/ No. 02

Pages: $78-88$

https://www.irojournals.com/tcsst/

DOI: https://doi.org/10.36548/jtcsst.2020.2.002

processing and analysing the data are mostly done in fog devices which are close and near to IoT devices and reduced transferring of data to cloud data centres. Reduction in network usage helps to minimize the budget for network bandwidth upgradation and lessen the bandwidth congestion problem.

\section{Conclusion}

This research work has designed and simulated a fog based smart surveillance system in iFogsim and performed a comparative study on the performance of the Cloud only and Fog-Based Cloud Network approach in different configurations. The results of the simulation are embraced to determine the performance highlights of the Fog-Based Cloud Network over Cloud Only Network approach. The results of the experiment showed that application loop delay is reduced, energy consumption of the device is lower, execution cost is efficient, and network usage is fairly lesser in Fog-Based Cloud Network approach than that in Cloud Only Network approach. As a result, real time security surveillance system is improved in Fog-Based Cloud Network approach. Fog Computing is a new paradigm and an extension of Cloud Computing. This better performance results justifies the suitability of IoT applications using Fog-Based Cloud Network approach.

\section{References}

[1] Bellavista, P., Berrocal, J., Corradi, A., Das, S. K., Foschini, L., \& Zanni, A. (2019). A survey on fog computing for the Internet of Things. Pervasive and Mobile Computing, 52, 71-99. https://doi.org/10.1016/j.pmcj.2018.12.007

[2] Buyya, R. (2018). Modelling and Simulation of Fog and Edge Computing Environments using iFogSim Toolkit Redowan Mahmud and Rajkumar Buyya. (April), 1-35.

[3] Byrne, J., Svorobej, S., Giannoutakis, K. M., Tzovaras, D., Byrne, P. J., Östberg, P.O., ... Lynn, T. (2017). A Review of Cloud Computing Simulation Platforms and Related Environments. (Closer), 679-691. https://doi.org/10.5220/0006373006790691

[4] Dastjerdi, A. V., Gupta, H., Calheiros, R. N., Ghosh, S. K., \& Buyya, R. (2016). Fog Computing: Principles, architectures, and applications. Internet of Things: Principles and Paradigms, 61-75. https://doi.org/10.1016/B978-0-12-805395-9.00004-6

[5] Gupta, H., Vahid Dastjerdi, A., Ghosh, S. K., \& Buyya, R. (2017). iFogSim: A toolkit for modeling and simulation of resource management techniques in the Internet of Things, Edge and Fog computing environments. Software - Practice and Experience, 47(9), 1275-1296. https://doi.org/10.1002/spe.2509

[6] Jamil, B., Shojafar, M., Ahmed, I., Ullah, A., Munir, K., \& Ijaz, H. (2020). A job scheduling algorithm for delay and performance optimization in fog computing. Concurrency Computation , 32(7), 1-13. https://doi.org/10.1002/cpe.5581

[7] Mahmoud, M. M. E., Rodrigues, J. J. P. C., Saleem, K., Al-Muhtadi, J., Kumar, N., \& Korotaev, V. (2018). Towards energy-aware fog-enabled cloud of things for healthcare. Computers and Electrical Engineering, 67, 58-69. https://doi.org/10.1016/j.compeleceng.2018.02.047

[8] Nakamura, S., Duolikun, D., Oma, R., Enokido, T., \& Takizawa, M. (2018). An 
Journal of trends in Computer Science and Smart technology (TCSST) (2020)

Vol.02/ No. 02

Pages: $78-88$

https://www.irojournals.com/tcsst/

DOI: https://doi.org/10.36548/jtcsst.2020.2.002

energy-efficient model for fog computing in the Internet of Things (IoT). Internet of Things, 1-2, 14-26. https://doi.org/10.1016/j.iot.2018.08.003

[9] Rahbari, D., \& Nickray, M. (2019). Low-latency and energy-efficient scheduling in fog-based IoT applications. Turkish Journal of Electrical Engineering and Computer Sciences, 27(2), 1406-1427. https://doi.org/10.3906/elk-1810-47

[10] Shi, C., Ren, Z., Yang, K., Chen, C., Zhang, H., Xiao, Y., \& Hou, X. (2018). Ultra-low latency cloud-fog computing for industrial Internet of Things. IEEE Wireless Communications and Networking Conference, WCNC, 2018-April, 1-6. https://doi.org/10.1109/WCNC.2018.8377192

[11] Sucharitha, V., Prakash, P., \& Iyer, G. N. (2019). Agrifog-a fog computing based IoT for smart agriculture. International Journal of Recent Technology and Engineering, 7(6), 210-217.

[12] Svorobej, S., Takako Endo, P., Bendechache, M., Filelis-Papadopoulos, C., Giannoutakis, K., Gravvanis, G., ... Lynn, T. (2019). Simulating Fog and Edge Computing Scenarios: An Overview and Research Challenges. Future Internet, 11(3), 55. https://doi.org/10.3390/fi11030055

[13] Yousefpour, A., Ishigaki, G., \& Jue, J. P. (2018). Fog Computing: Towards Minimizing Delay in the Internet of Things. Springer International Publishing AG, (c), 87-115. https://doi.org/10.1007/978-3-319-57639-8

\section{Authors Biography}

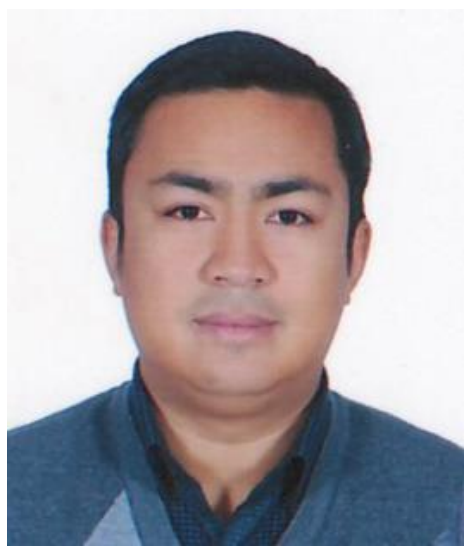

SUJAN SHRESTHAis pursuing his final semester of M. Phil in ICT at Faculty of Science, Health and Technology, Nepal Open University, Lalitpur Nepal.He has received the Bachelor of Technology degree in Electronics and Communication Engineering from National Institute of Technology, Durgapur, India, in 2005. He received the Master of Science in Information and Communication Engineering from Pulchowk Campus, Institute of Engineering, Lalitpur, Nepal in 2010. He works in Nepal Telecomin the capacity of Senior Engineer (Telecom) and has been working in Nepal Telecom since 2007.

Hisareas of research interests include Artificial Intelligence, Machine Learning, Deep Learning, Big Data Analytics, Cloud/Fog Computing, Computer Vision Technology, and Natural Language Processing. 
Journal of trends in Computer Science and Smart technology (TCSST) (2020)

Vol.02/ No. 02

Pages: $78-88$

https://www.irojournals.com/tcsst/

DOI: https://doi.org/10.36548/jtcsst.2020.2.002

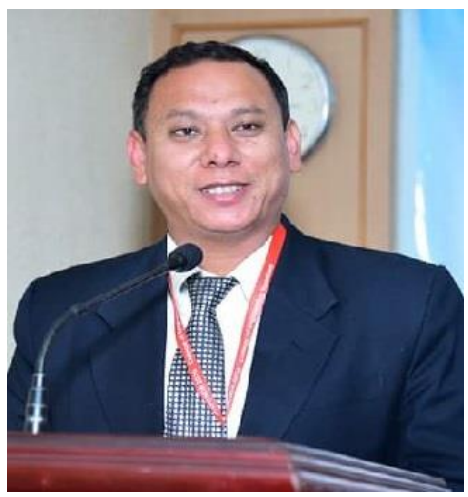

SUBARNA SHAKYA has received the MSc and PhD degrees in Computer Engineering from the Lviv Polytechnic National University, Ukraine, 1996 and 2000 respectively. He is the Professor of Computer Engineering, Department of Electronics and Computer Engineering, Pulchowk Campus, Institute of Engineering, Pulchowk, Tribhuvan University, Nepal. He has served as Executive Director at National Information Technology Centre, Government of Nepal.

His areas of research interests include E-Government system, Computer Systems \& Simulation, Cloud computing \& security, Software Engineering \& Information System, Computer Architecture, Multimedia system. 